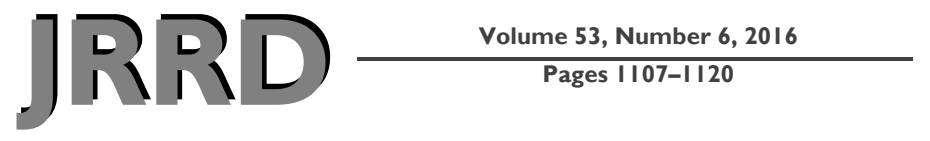

\title{
Preliminary evaluation of a novel bladder-liner for facilitating residual- limb fluid volume recovery without doffing
}

\author{
Joan E. Sanders, PhD; " Christian B. Redd, MS; John C. Cagle, BS; Brian J. Hafner, PhD; David Gardner, \\ MSME; Katheryn J. Allyn, CPO; Daniel S. Harrison, BS; Marcia A. Ciol, PhD \\ Department of Bioengineering, University of Washington, Seattle, WA
}

\begin{abstract}
For people who wear a prosthetic limb, residuallimb fluid volume loss during the day may be problematic and detrimentally affect socket fit. The purpose of this research was to test the capability of a novel liner with adjustable bladders positioned within its wall to mitigate volume loss and facilitate limb fluid volume recovery and retention. Bioimpedance analysis was used to monitor fluid volume changes in the anterior and posterior residual limb of participants with transtibial amputation. Participants underwent six cycles of sitting for $90 \mathrm{~s}$, standing for $90 \mathrm{~s}$, and walking for $5 \mathrm{~min}$ with liquid within the bladder-liners. Between the third and fourth cycles, participants sat for 10 min with liquid left within the bladders (Liquid-In) or removed (Liquid-Out). Results showed that participants recovered more fluid volume during the 10 min of sitting with Liquid-Out than Liquid-In ( $p=0.09$ for anterior and $p=0.04$ for posterior). However, those fluid volume recoveries were not well retained in the short term (after the fourth cycle) or the long term (after the sixth cycle). Physiologic differences between sessions, reflected in the rates of fluid volume change at the outset of the session, and excessive stiffness of the bladder-liners may have affected fluid volume retentions.
\end{abstract}

Key words: accommodation, amputation, bioimpedance, bladder liquid, limb loss, prosthetic limb, residual limb, retention, transtibial amputation, volume.

\section{INTRODUCTION}

People who wear a prosthetic limb have significant problems with residual limb volume loss during the day.
Even small changes in limb volume can affect changes in socket fit that, if not corrected, can lead to soft tissue injury. Importance of the limb-to-socket volume relationship was demonstrated in a study on 10 participants with transtibial amputation, in which socket volume reductions as small as 1.0 percent induced clinically detectable alterations in socket fit [1].

There are, in general, two basic methods to accommodate limb volume change: adjust the size of the prosthesis (e.g., socket or interface) or adjust the size of the residual limb. The first is considered an extrinsic approach to limb volume management and the second an intrinsic approach. The most common means for adjusting the prosthesis is to add or remove prosthetic socks. Though sock addition or removal can generally accommodate limb volume changes, it has three important limitations. First, the person with limb loss must sense that sock addition or removal is necessary. Sensation is often impaired in people with limb loss, and this impairment may adversely affect their ability to know when a sock change is needed [2]. Second, the prosthesis must be doffed to add socks. Socket doffing can be embarrassing

\footnotetext{
Abbreviation: SD = standard deviation.

*Address all correspondence to Joan E. Sanders, PhD; Bioengineering 355061, Foege N430J, 3720 15th Ave NE, University of Washington, Seattle WA 98195; 206-221-5872; fax: 206-685-3300.

Email: janders@u.washington.edu

http://dx.doi.org/10.1682/JRRD.2014.12.0316
} 
or inconvenient, particularly if clothing (e.g., long pants) needs to be removed. Third, adding socks may cause the residual limb to further reduce in volume [3]. Thus, even though sock addition is an extrinsic strategy to volume management, it may have an intrinsic effect on the residual limb.

The most common means to change the size of the residual limb is to increase vacuum within the socket, typically through the use of a suction socket or an elevated vacuum device. Increasing vacuum within the socket presumably causes fluid to be drawn into the residual limb during the swing phase of gait [4]. Vacuum has been demonstrated to enhance limb volume response in some studies [4-7] but not others [8-10]. Reports on skin response to elevated vacuum have also been mixed, with some investigators reporting benefits [11-13] and others reporting skin injury [14]. In elevated vacuum studies that showed mixed results, factors such as quality of the socket fit or presence of comorbidities may have affected the elevated vacuum system's ability to maintain residual-limb fluid volume. How socket fit, user health, and other factors interact and affect limb volume is not well understood.

Another means to intentionally adjust the size of the residual limb, and one that is not often utilized, is to temporarily doff the prosthesis. Doffing the prosthesis reduces interstitial fluid pressures, facilitating higher arterial-tointerstitial transport and thus inducing limb fluid volume recovery [15]. While doffing may effectively facilitate volume recovery, it can be inconvenient to perform. Further, accidently doffing for too long may induce edema for some prosthesis users and make redonning the prosthesis difficult and painful.

The purpose of this research was to evaluate a new technology to facilitate fluid volume recovery and retention that does not require prosthesis doffing or fabrication of a suction or vacuum socket. We termed the technology a "bladder-liner." A bladder-liner has adjustable bladders positioned within the liner's elastomeric wall (Figure 1). Tubes exit at the proximal end of the liner so that liquid can be easily added or removed. The bladders can be filled with liquid, thereby reducing volume of the socket and interface and enhancing limb-socket mechanical coupling. Removing liquid reduces liner volume and loosens the prosthesis, relieving pressures and potentially allowing residual-limb fluid volume recovery. In this initial effort, we conducted preliminary in-laboratory studies to determine if the bladder-liners had a significant effect on fluid

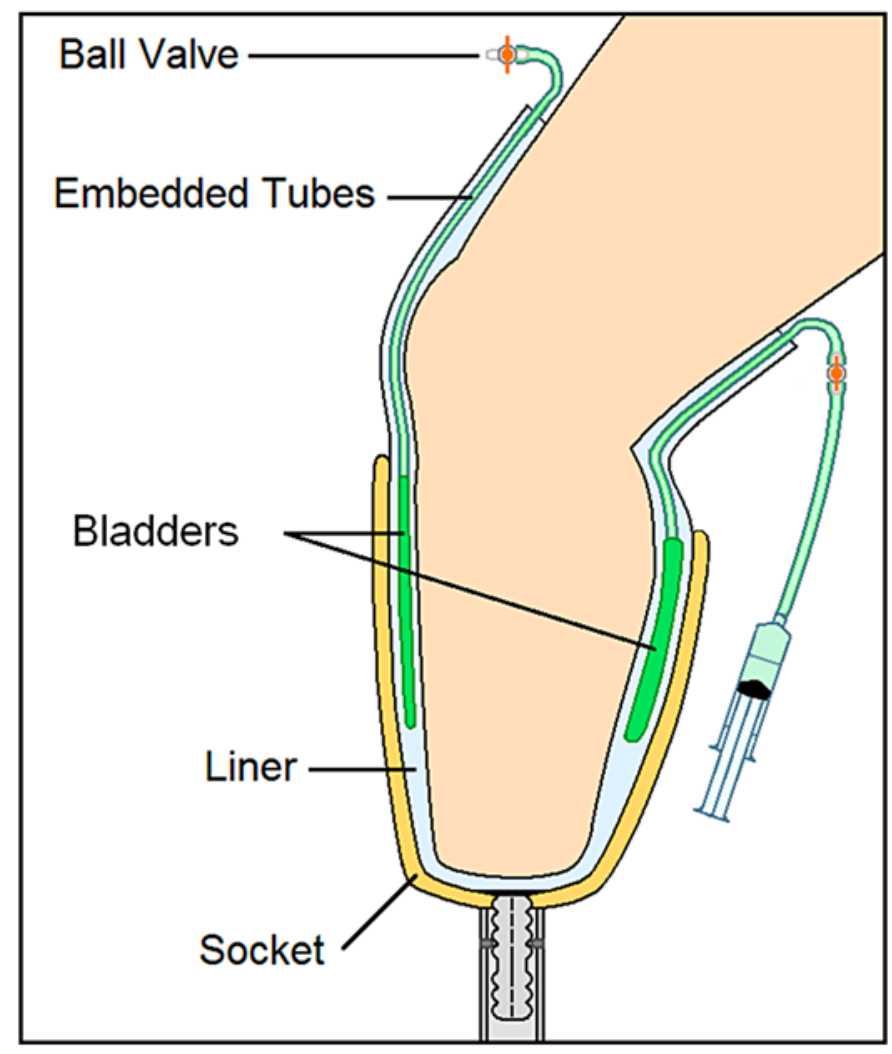

Figure 1.

Bladder-liner used in this research. Bladders were embedded within the liner wall. Polymer tubes of $2.4 \mathrm{~mm}$ outer-diameter, also embedded within the liner, extended from the proximal edge to a detachable syringe.

volume recovery. We tested the hypothesis that removing liquid from the bladder-liner during resting after walking would affect limb fluid volume recovery. More specifically, we tested the hypothesis that the median difference in limb fluid volume during resting with liquid released from the bladder-liners, compared with liquid not released from the bladder-liners, was not zero. We did not pose a directional hypothesis because previously we found some participants increased in limb fluid volume after doffing the socket and some decreased [15]. We also tested the hypothesis that recovered fluid would be well maintained during subsequent periods of activity. More specifically, we tested the hypothesis that the median difference in volume retention after resting with liquid released from the bladder-liners, compared with liquid not released, was not zero. Because the studies were short term, we were able to control variables that may have affected limb fluid volume (i.e., the type and duration of activity, the walking surface, 
and weight-bearing levels during standing). If the hypotheses were supported by the data, then use of a bladder-liner for limb volume management would be demonstrated.

\section{METHODS}

\section{Participants}

Volunteers were considered for inclusion if they had a transtibial amputation more than 12 mo prior and were at a Medicare Functional Classification Level of K2 or higher (at least a limited community-level ambulator). Participants also needed to be using a properly fitted prosthesis, as evaluated by the research prosthetist. To assess socket fit, the research prosthetist carefully examined the participant's residual limb and asked about his or her health and prosthesis history. Socket fit was considered acceptable if the residual limb was free of sores or abrasions, the socket was not undergoing revision by the participant's prosthetist, and the participant reported that the socket was comfortable. Participants were excluded if they demonstrated neuropathy to the point that they could not feel when liquid was added to the bladders. Participants' normal liner sizes needed to be approximately equivalent to the bladder-liner created for this study (described subsequently). Participants were required to have sufficient room in their socket for the bladder-liner (i.e., they regularly wore at least one prosthetic sock in addition to their normal liner). Because we intended to generalize results to people who use prostheses on a daily basis, participants needed to report regularly using their prosthesis at least $8 \mathrm{~h} / \mathrm{d}$. Participants were required to be capable of completing the study protocol, which included 5 min of continuous treadmill walking as well as standing with equal weight-bearing for at least $90 \mathrm{~s}$. Exclusion criteria included use of an assistive device (e.g., cane, walker), injuries or skin breakdown on the residual limb, presence of peripheral neuropathy determined by microfilament testing, or metal implants within the affectedside limb. Use of an assistive device with presence of skin issues may have put participants at risk for harm, and metal implants could distort bioimpedance measurement of limb fluid volume. Participants were excluded if their residual limb length was less than $5.5 \mathrm{~cm}$, as this was the distance needed between voltage-sensing electrodes.

\section{Bladder-Liner}

Bladder-liners were fabricated using a custom procedure developed by Sandia National Laboratories (Albuquerque, New Mexico) [16]. Because the bladders were intended to be used with the user's regular prosthesis, a new socket was not fabricated. The first step of the bladder fabrication procedure was to make positive and negative molds of a size medium commercial elastomeric liner (6 $\mathrm{mm}$ thickness). This liner size was selected because it is commonly prescribed to prosthetic limb users in our local area (Seattle, Washington) and would facilitate recruitment of potential participants. Three custom-made bladders, made from 100 percent platinum cure silicone, were installed in the space between the positive and negative mold and held in place with brackets. We selected platinum cure silicone because it is similar to material used in commercial elastomeric liners and it is compatible with the fabrication process. A $0.8 \mathrm{~mm}$ inner-diameter, $2.4 \mathrm{~mm}$ outer-diameter tube extended from the top of each bladder and was positioned in the space between molds. One-hundred percent platinum cure silicone was then injected into the space between the molds and allowed to dry. This procedure ensured that both the bladders and tubes were embedded within the liner wall.

Sandia National Laboratories performed bladder compression testing. Water-filled bladders were placed in a vice and a pressure gauge was connected to the tube exit. The bladders successfully tolerated fluid pressures to $250 \mathrm{kPa}$, which were the maximal pressures expected in the regions where the bladders were to be placed [17].

Bladder sizes were customized to each participant's limb dimensions and were located at load-bearing sites on the residual limb (i.e., along their anterior medial and anterior lateral tibial flares and along the posterior midline at midlength). The posterior bladder was slightly wider at the top than bottom so as to capture the triangular popliteal space. Anterior medial and anterior lateral bladders were the same shape, narrow and long, with an approximately uniform width. The anterior bladders ran longitudinally on either side of the tibial crest, distal to the tibial tuberosity, to approximately $2 \mathrm{~cm}$ proximal to the distal end of the tibia. Bladders were filled with colored water using syringes and luer locks and connected to the tubes exiting from the socket brim. Water was used because it is readily available, incompressible, and a primary component of a related commercial product (Active Contact System, Simbex; Lebanon, New Hampshire). The water was colored so that it could be seen easily within the syringe. 


\section{Limb Fluid Volume Measurement}

Limb fluid volume changes were measured using a custom bioimpedance analysis system and custom electrodes [18]. An embedded personal computer (Innovative Integration; Simi Valley, California) and peripherals (X3$25 \mathrm{M}$ and $\mathrm{X} 3-10 \mathrm{M} X \mathrm{XMC}$ IO modules) were configured into a multifrequency bioimpedance platform, which delivered bursts of alternating current stimulus to the limb, received voltage-sense signals from the limb, demodulated the voltage and current signals, displayed the data, and stored them to disk. We monitored data from two channels, one anterior and one posterior.

We used custom skin electrodes because existing commercial electrodes are not thin or durable enough for this application. We fabricated the electrodes using conductive tape (ARCare 8881, Adhesives Research Inc; Glen Rock, Pennsylvania); multistranded, silver-plated, copper wire with an aramid core and PVC insulation (New England Wire; Lisbon, New Hampshire); a flattened brass crimp to strain relieve wires as they exited the insulation within the electrode; and a very thin layer of hydrogel (ultrasonic coupling gel, GE Panametrics, NDT; Waltham, Massachusetts) between the electrode and skin.

The distance between electrodes varied because they were positioned relative to anatomical landmarks. Sweat did not influence bioimpedance results because the electrical current path was through the hydrogel and skin immediately beneath the conductive tape. The resistance of skin was too high for sweat outside the conductive tape to affect results.

Current-injection electrodes were positioned on the proximal thigh and the distal aspect of the residual limb to deliver tone bursts of sinusoidal electrical current $(\sim 300 \mu \mathrm{A})$ to the limb (Figure 2). The proximal thigh electrode was made up of two $15.0 \times 1.5 \mathrm{~cm}$ electrodes, one anterior and one posterior, centered on the midline of the limb. The current-injecting electrodes were positioned at least $20 \mathrm{~cm}$ from the most proximal voltagesensing electrode. The distal residual-limb electrode, of diameter $3.5 \mathrm{~cm}$, was positioned on the center of the distal surface. If bony prominences were present, we moved the distal electrode so that it was located over soft tissue but still equidistant from the anterior lateral and posterior aspects of the limb. Voltage-sensing electrode pairs were positioned on the anterior and posterior aspects of the residual limb. Each voltage-sensing electrode was of dimension $5.0 \times 1.5 \mathrm{~cm}$. The anterior electrodes were

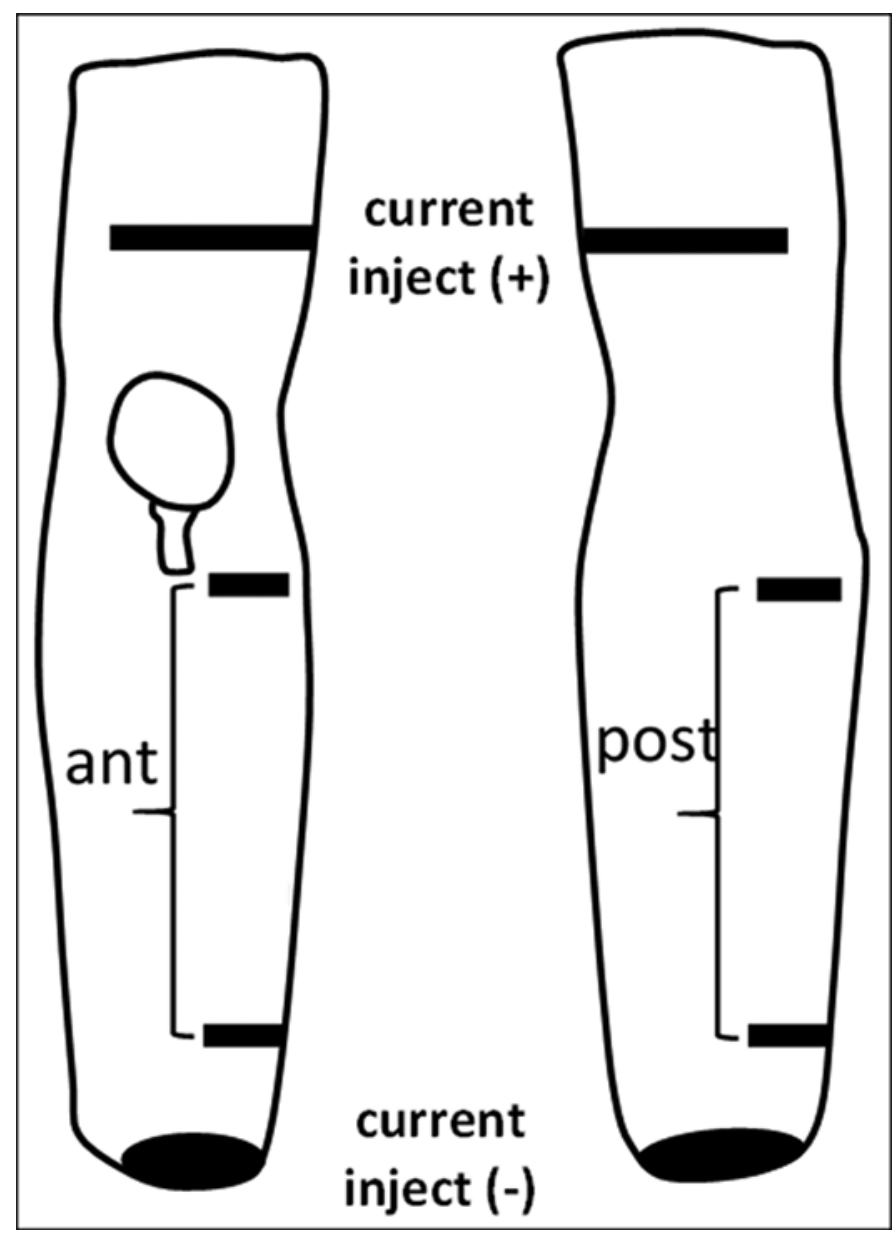

Figure 2.

Electrode positions on the residual limb: anterior (ant) and posterior (post) views. Current-injection electrodes were positioned on the proximal thigh and distal limb. Voltage-sensing electrodes ( 2 channels) were positioned on the anterior-lateral and posterior residual limb. Rectangular current injection electrodes $(15.0 \times 1.5 \mathrm{~cm})$ were placed on the proximal thigh, one on the anterior surface and one on the posterior surface. A circular current injection electrode (3.5 cm diameter) was positioned on the distal residual limb. The proximal voltage-sensing electrodes $(5.0 \times 1.5 \mathrm{~cm})$ were placed at the level of the patellar tendon, and the distal voltage-sensing electrodes were placed just proximal to the level of the distal end of the tibia and a minimum of $4.5 \mathrm{~cm}$ from the distal current injecting electrode.

located over the anterior lateral muscle group. The posterior electrodes were positioned over the central posterior muscle group. The proximal voltage-sensing electrodes were positioned at the level of patellar tendon, distal to the fibular head. The distal voltage-sensing electrodes 
were positioned such that their lower edges were at the level of the distal tibia. Bursts at each of 24 frequencies were delivered at a rate of 20 sets of bursts per second $(20 \mathrm{~Hz})$. The bursts were logarithmically distributed from $5 \mathrm{kHz}$ to $1 \mathrm{MHz}$. The custom bioimpedance instrument sensed and demodulated the current-injected and voltagesensed signals, determined average real and imaginary components for each tone burst, and stored the results to disk. The system had 16-bit resolution. Data were postprocessed using de Lorenzo's form of the Cole model [19] to determine extracellular fluid impedance. A limb segment model [20] was then used to convert extracellular fluid impedances to extracellular fluid volumes:

$$
V_{E C F}=\frac{1}{1000}\left(\frac{\rho_{E C F} C}{R_{E C F}}\right)^{\frac{2}{3}} \frac{L^{\frac{5}{3}}}{(4 \pi)^{\frac{1}{3}}}
$$

where $V_{E C F}$ was extracellular fluid volume, $\rho_{E C F}$ was specific resistivity of the extracellular fluid, $C$ was midlimb circumference, $R_{E C F}$ was extracellular fluid resistance, and $L$ was length between the electrodes. In this study, we investigated only extracellular fluid volume changes and not intracellular changes because extracellular changes were expected to be most relevant to the relatively shortterm limb fluid volume fluctuations measured in this study.

We tested the electrodes to determine if liquid presence within the bladders affected the electrical performance of the bioimpedance system. A benchtop test system was constructed using a garolite base plate with four silver contact pads for each electrode: positive current injection, negative current injection, positive voltage sense, and negative voltage sense. We tested each of the two voltage-sense channels of the instrument separately. A reactive bioimpedance test circuit with component values comparable to those recorded on participants with amputation (i.e., extracellular resistance $48.7 \Omega$, intracellular resistance $158.0 \Omega$, and membrane capacitance $10 \mathrm{nF}$ ) was tested. While bioimpedance data were being collected, an empty bladder was placed in between and on top of the voltage sense electrodes. The bladder was filled with $30 \mathrm{~mL}$ of water and then emptied. Impedances measured with the bladder on the electrodes before filling, after filling, and after emptying were compared with those measured before placing the bladder on the electrodes.

\section{Testing Protocol}

Each participant visited the laboratory three times, once to fit the bladder-liner and twice for bioimpedance testing.

Liquid volumes appropriate for each participant were established during the bladder-liner fitting session. Adjustment of anterior bladders tended to affect load distributions in tissues within the midsection of the residual limb. Filling the posterior bladders caused participants to feel "lifted" in their socket and prevented them from sinking deeper. The research practitioner typically started the fitting session by filling the posterior bladder to get participants to their right "height" in the socket, and then filling the anterior bladders to fine tune the load distribution. Some participants preferred equal amounts of liquid in the anterior bladders while others preferred unequal amounts.

Liquid was inserted in $2.5 \mathrm{~mL}$ to $7.0 \mathrm{~mL}$ increments into each bladder. We selected this range because it was used effectively in pilot testing of the bladder-liners. The syringe delivering the liquid had a resolution of $0.5 \mathrm{~mL}$. After each increment the participant sat, stood, and walked to evaluate comfort and then reported his or her comfort rating to the research practitioner. This procedure was repeated, adding additional increments of liquid in each of the bladders until the participant reported discomfort or the research practitioner considered the configuration unsafe for the participant. Then liquid was removed in $2.5 \mathrm{~mL}$ to $7.0 \mathrm{~mL}$ increments until the participant reported an optimal fit. With the bladders filled at the optimal liquid volumes, the participant walked on the treadmill and determined his or her self-selected walking speed.

The same liquid volumes that were determined for each participant in the initial bladder-liner fitting session were used in the bioimpedance test sessions. There were only two states: the bladders were filled or they were empty.

The first bioimpedance test session was scheduled to take place within 1 wk of the bladder-liner fitting session, with the second bioimpedance test session at least $2 \mathrm{~d}$, but not more than $5 \mathrm{wk}$ after that. Sessions were scheduled at the same time of day in an effort to minimize time-of-day differences on the results. The two bioimpedance test sessions were identical, except that in one session, bladder liquid was removed during a $10 \mathrm{~min}$ sitting period in the middle of the test (termed "LiquidOut"), while in the other session it was not (termed "Liquid-In"). We randomly selected the order of which protocol was run first, Liquid-In or Liquid-Out. 
In both sessions, participants entered the laboratory and then sat with good posture in a chair for a $10 \mathrm{~min}$ period to achieve a homeostatic condition. During this time, the research practitioner queried the participant about recent changes made to his or her prosthesis, recent activity, and overall health. The researcher cleaned the participant's residual limb with Tracer Prep (3M; St. Paul, Minnesota) at sites where electrodes were to be placed and then instrumented the participant with the custom bioimpedance electrodes (Figure 2). Electrodes were covered with Tegaderm (3M) to ensure edges did not peel up during testing. Wires exited at the lateral posterior aspect of the socket, extending under the participant's clothing and connecting to an instrument box (360 g) on a belt worn around the waist. A $305 \mathrm{~cm}$ cable extended from the instrument box to a stationary custom bioimpedance system [18] (Figure 3).

We initiated data collection once we placed bioimpedance electrodes on the residual limb at the same positions and spacing used in the fitting session. The participant donned the liner and the prosthesis. The bladders were then filled with liquid to the previously determined optimum amount, and the participant was asked to stand and weight-shift briefly to ensure the prosthesis was properly donned. The participant then underwent three 8.1 min cycles of sitting for $90 \mathrm{~s}$, standing with equal weight-bearing for $90 \mathrm{~s}$, walking on a treadmill for $5 \mathrm{~min}$,

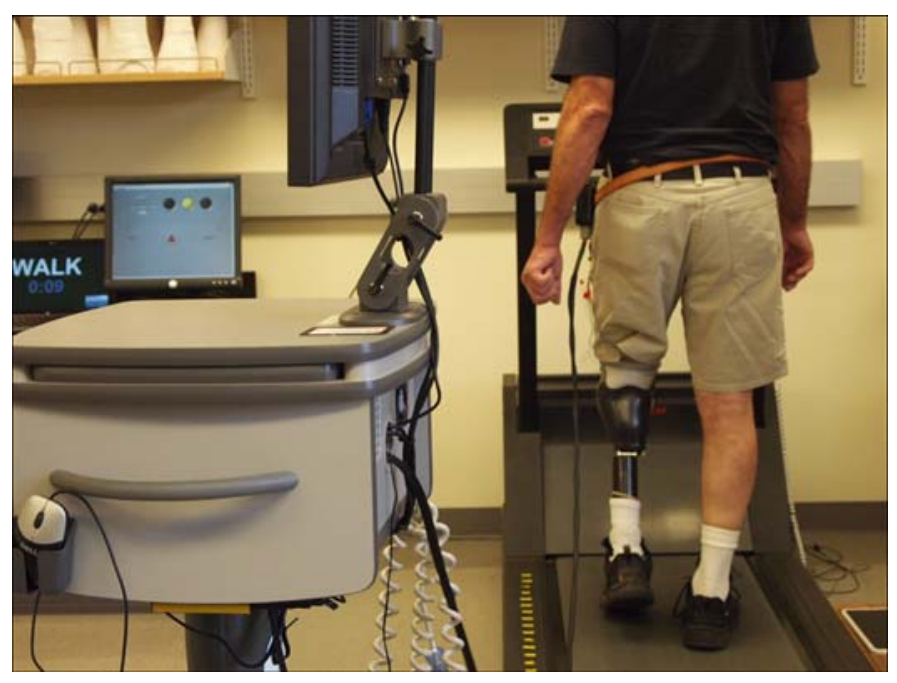

Figure 3.

Data collection session. The participant wore a waist-belt box tethered to a stationary bioimpedance system. and standing with equal weight-bearing for $5 \mathrm{~s}$. We used these durations because prior studies demonstrated that $90 \mathrm{~s}$ stands and 5 min walks were consistent with activity patterns in active prosthesis users [15]. The treadmill was set at the participant's self-selected walking speed. After the third cycle $5 \mathrm{~s}$ standing segment was completed, the participant sat with good posture in a stable chair for 10 min. For the Liquid-In session, no changes were made to the bladders during the 10 min sitting period. For the Liquid-Out session, bladder liquid was removed right after the participant sat down by connecting a large syringe to all three bladder tubes simultaneously and drawing out the liquid, a process that took approximately $25 \mathrm{~s}$. We measured the extracted liquid volume to ensure that all liquid was removed. At the end of the $10 \mathrm{~min}$ period in the Liquid-Out session, the liquid was returned to the bladders. The participant conducted three additional cycles of sitting for $90 \mathrm{~s}$, standing for $90 \mathrm{~s}$, walking on a treadmill for $5 \mathrm{~min}$, and standing with equal weightbearing for $5 \mathrm{~s}$.

\section{Data Processing and Analysis}

Bioimpedance analysis on participants' residual limbs may be sensitive to skin strains induced by participants' postures. Skin strains may alter the distance between electrodes. This change would not be accounted for in the models used to convert raw data to limb fluid volume because a consistent electrode spacing is assumed. Thus, an error might be introduced to the measurement. We therefore compared differences in fluid volumes only for like postures (e.g., standing with equal weight-bearing or sitting with feet flat on the floor). This methodology ensured consistent knee flexion and associated skin strains between the voltage-sensing electrodes.

Limb fluid volume changes were calculated during the brief stand after each walk cycle (i.e., six data points) and during sitting at the beginning and end of the 10 min resting phase between the third and fourth cycles (i.e., two additional data points). Liquid was not within the bladders for the resting calculations for Liquid-Out. This methodology ensured that there were no posture differences between data points subtracted from each other, which otherwise might have affected limb fluid volume results. The limb fluid volume after the third cycle $\left(V_{\text {postwalk3 } 3}\right)$ was used as the reference volume because the two bioimpedance test session 
protocols were identical up to that point. The following calculations were performed:

\section{Rest Period Percentage Fluid Volume Change}

$$
=\left[\frac{\left(V_{\text {endrest }}-V_{\text {begrest }}\right)}{V_{\text {postwalk } 3}}\right] \times 100 \%
$$

\section{Short-Term Percentage Fluid Volume Retention}

$$
=\left[\frac{\left(V_{\text {postwalk } 4}-V_{\text {postwalk } 3}\right)}{V_{\text {postwalk } 3}}\right] \times 100 \%
$$

Long-Term Percentage Fluid Volume Retention

$$
=\left[\frac{\left(V_{\text {postwalk } 6}-V_{\text {postwalk } 3}\right)}{V_{\text {postwalk } 3}}\right] \times 100 \%
$$

where $V_{\text {endrest }}$ was fluid volume during sitting at the end of the rest period, $V_{\text {begrest }}$ was fluid volume during sitting at the beginning of the rest period, and $V_{\text {postwalk(n) }}$ was fluid volume during the brief stand after the $n$th walk cycle.

We conducted Wilcoxon sign-rank tests for paired data to assess whether there was a difference between medians of Liquid-Out versus Liquid-In conditions for the following measures: (1) rest period percentage fluid volume change, (2) short-term percentage fluid volume retention, and (3) long-term percentage fluid volume retention. We used this nonparametric test that relied on rank because the sample size was small and the specific distribution of the data was not known. Data from anterior and posterior channels were considered separately. Analyses were performed using SPSS version 23.0 (IBM; Armonk, New York). All hypotheses were evaluated with a threshold for significance of 0.05 . To limit the potential for type II error at this exploratory stage of research, we did not perform adjustments for multiple comparisons [21].

We also calculated a rate of fluid volume change during the initial part of the session, using fluid volumes measured during the brief stands after the third walk cycle and after the second walk cycle. We did this because we anticipated that session-to-session differences existed. In other words, we expected that some days participants entered the laboratory experiencing a high limb fluid volume loss rate (high loss rate day) and on other days a low limb fluid volume loss rate (low loss rate day), independent of our testing protocol. Such differences may have been dictated by activity prior to entering the laboratory, recent food and liquid intake, or other variables, and they might have affected our results. For example, a person might experience a different response to the intervention on a high loss rate day, compared with a low loss rate day. If this result were demonstrated, then it would point to the effect a participant's day-to-day physiological variability has on accommodation system performance and the need to account for such variability in the design of the system. We used the brief stands after the third walk cycle and after the second walk cycle as time points because the testing protocol was the same up to the brief stand after the third walk cycle. We plotted results of fluid volume versus time and then investigated whether volume retentions were greater for the condition (Liquid-In or Liquid-Out) with the higher rate of fluid volume change.

\section{RESULTS}

Mean bench testing results for differences between measurements taken with the bladder filled versus empty \pm standard deviation (SD) were $0.004 \pm 0.002$ percent. The absolute value of the differences was $0.006 \pm 0.005$ percent.

Of the eight participants in this study, seven had unilateral amputation and one had bilateral amputations. Seven participants were male and one was female. The average age of participants \pm SD was $51 \pm 14 \mathrm{yr}$ and $15 \pm 9 \mathrm{yr}$ since limb amputation. Mean residual-limb length \pm SD was $19 \pm$ $3 \mathrm{~cm}$, and mean midlength circumference was $29 \pm 2 \mathrm{~cm}$. Two participants had comorbidities: two had peripheral arterial disease, and one of those individuals had a heart condition for which he took medications that may have affected his limb fluid volume. However, his medications were the same in both sessions. Additional participant details are provided in Table 1.

Start times of the sessions ranged from 8:50 am to 2:10 pm; the mean start time \pm SD was 11:30 am $\pm 2.0 \mathrm{~h}$ and the median was 10:30 am. The two test sessions for each participant were conducted at approximately the same time of day. Start time differences between sessions ranged from 10 to 110 min with a mean $\pm \mathrm{SD}$ of $50 \pm 40 \mathrm{~min}$ and a median of $40 \mathrm{~min}$.

The bladder-liner technology was well received by the participants. All participants wore the liners over the 
Table 1.

Participant characteristics.

\begin{tabular}{|c|c|c|c|c|c|c|c|c|c|}
\hline \multirow{2}{*}{ Characteristic } & \multirow{2}{*}{ Mean \pm SD } & \multicolumn{8}{|c|}{ Participant } \\
\hline & & 1 & 2 & 3 & 4 & 5 & 6 & $7^{*}$ & 8 \\
\hline$\overline{\text { Age (yr) }}$ & $51 \pm 14$ & 59 & 58 & 33 & 75 & 49 & 37 & 43 & 53 \\
\hline Cause of Amputation & - & Trauma & Trauma & Trauma & Trauma & $\begin{array}{l}\text { Trauma then } \\
\text { infection }\end{array}$ & Trauma & Trauma & Trauma \\
\hline $\begin{array}{l}\text { Time Since } \\
\text { Amputation (yr) }\end{array}$ & $15 \pm 9$ & 6 & 9 & 15 & 13 & 17 & 3 & 27 & 29 \\
\hline Mass (kg) & $91 \pm 11$ & 85 & 94 & 110 & 97 & 97 & 94 & 76 & 77 \\
\hline $\begin{array}{l}\text { Midlength } \\
\quad \text { Circumference }(\mathrm{cm})\end{array}$ & $29 \pm 2$ & 29 & 28 & 33 & 30 & 30 & 31 & 27 & 29 \\
\hline Sex & - & M & M & $\mathrm{F}$ & M & M & M & M & M \\
\hline Activity (h/d) & $14.9 \pm 1.2$ & 16.0 & 16.0 & 13.5 & 16.0 & 13.5 & 16.0 & 13.5 & 15.0 \\
\hline Prosthesis Design & - & РTB & $\begin{array}{l}\text { PTB Endo } \\
\text { TSB }\end{array}$ & РTB & $\begin{array}{l}\text { PTB and Pelite } \\
\text { Liner }\end{array}$ & $\begin{array}{l}\text { TSB Seal-In } \\
\text { Liner, Valve }\end{array}$ & TSB & РTB & РTB \\
\hline
\end{tabular}

${ }^{*}$ Subject with bilateral amputation.

$\mathrm{F}=$ female, $\mathrm{M}=$ male, $\mathrm{PAD}=$ peripheral arterial disease, $\mathrm{PTB}=$ patellar tendon bearing, $\mathrm{SD}=$ standard deviation, $\mathrm{TSB}=$ total surface bearing.

course of the test sessions without discomfort. Several participants made unsolicited comments that the bladderliner was more comfortable than their regular prosthetic liner. Interestingly, participants were not interested in adjusting bladder liquid volume on different days, and instead were satisfied with the familiar socket shape induced using their previously established bladder liquid volumes.

One participant (\#5) experienced a bladder-liner failure that required it to be refabricated. The failure was a delamination between the bladder and liner. Because there was an extended time period before the participant was retested, bladder volumes for the new liner were determined for this participant the day of the first bioimpedance data collection session. All remaining participants were tested in accordance with the stated protocol.

Bladder liquid volumes varied across participants (Figure 4). Liquid amounts in individual bladders ranged from 2.5 to $30.0 \mathrm{~mL}$, with a median of $15.0 \mathrm{~mL}$. Total bladder liquid volume ranged from $27.5 \mathrm{~mL}$ to $75.0 \mathrm{~mL}$, with a median of $43.5 \mathrm{~mL}$. Between 95 and 100 percent of bladder liquid inserted at the outset of the test session was removed during the sitting period in each Liquid-Out test session.

Figure 5 shows the volume change given by LiquidOut - Liquid-In for each participant within each condition (rest period, short- and long-term retention) by site (anterior

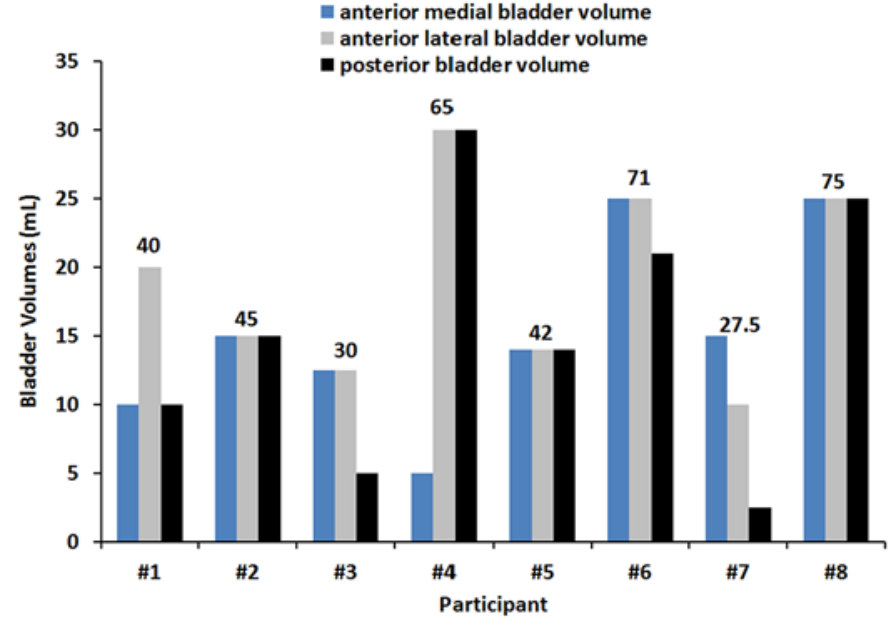

Figure 4.

Bladder volumes for each subject. Numbers above bars are total of all three bladder liquid volumes.

and posterior). Positive values represent a larger gain or a smaller loss for the Liquid-Out condition when compared with Liquid-In. Testing of the hypothesis that the median difference (Liquid-Out - Liquid-In) was not zero showed that during the resting period, the median difference change was 0.74 percent $(p=0.09$ for the test that the median was not 0 ) for anterior and 0.65 percent for posterior $(p=0.04)$. For short-term retention, median difference changes were 


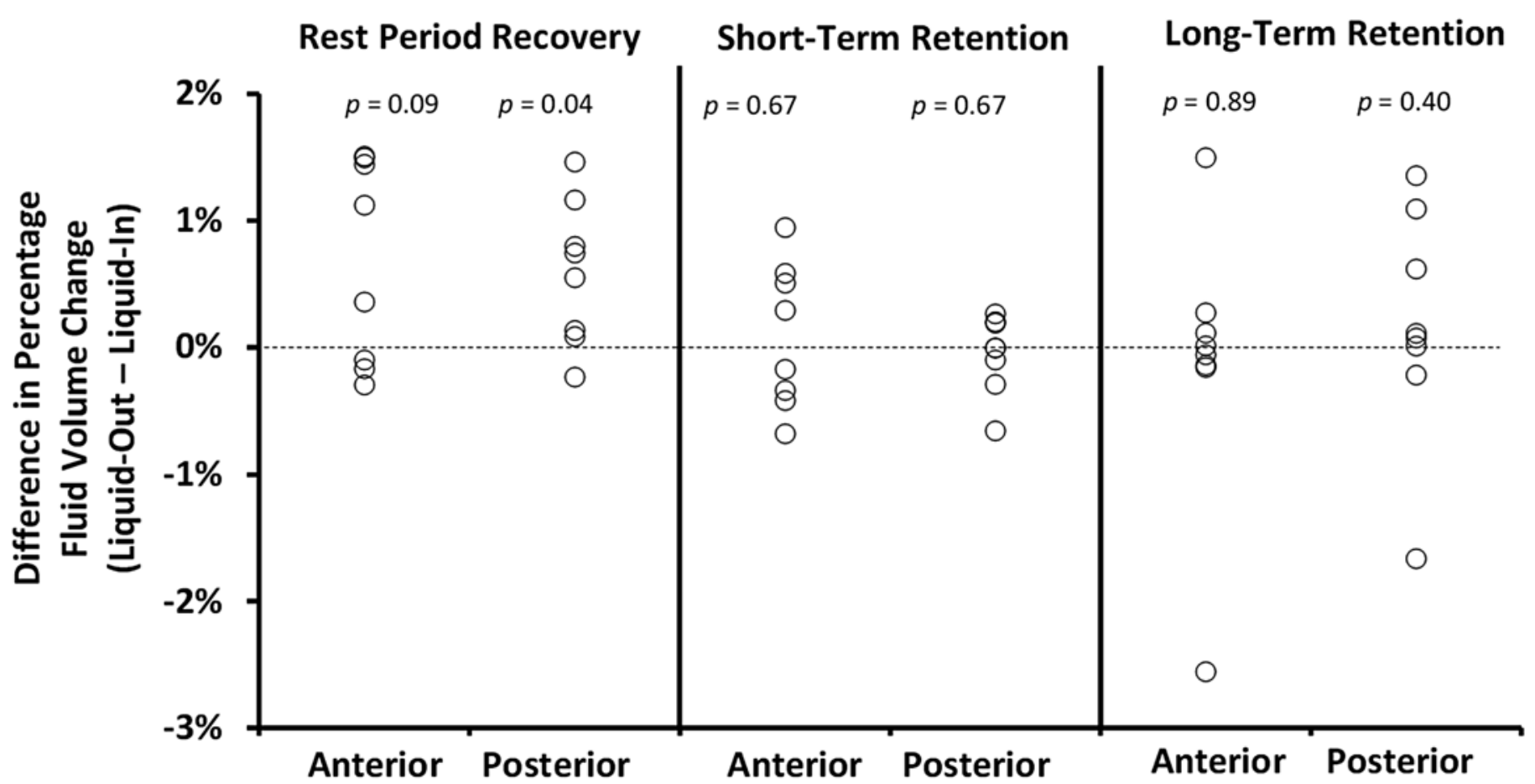

Figure 5.

Fluid volume changes for Liquid-Out versus Liquid-In. Rest period recovery, short-term retention, and long-term retention results. Medians are compared between Liquid-In and Liquid-Out conditions.

0.06 percent $(p=0.67)$ for anterior and 0.00 percent $(p=0.67)$ for posterior. For long-term retention, they were -0.02 percent $(p=0.89)$ and 0.09 percent $(p=0.40)$ for anterior and posterior, respectively.

Short-term and long-term percentage fluid volume retentions varied among participants. Four of the eight participants demonstrated greater anterior short-term percentage fluid volume retentions for Liquid-Out than Liquid-In. Similarly, five of eight participants showed greater posterior fluid volume retentions in the Liquid-Out condition. Long-term percentage fluid volume retentions were greater for Liquid-Out than Liquid-In at anterior sites for four of eight participants and at posterior sites for five of eight participants.

Visual inspection of individual participants' data revealed that the rate of fluid volume change before the rest period was not consistent between test sessions (Figure 6). Rates of change ranged from -9.2 to 6.4 percent per hour (mean $=-1.2 \%$ per $\mathrm{h}$, median $=-1.0 \%$ per $\mathrm{h}$ ) for anterior sites and -14.6 to 11.2 percent per hour (mean $=-1.1 \%$ per $\mathrm{h}$; median $=-1.3 \%$ per hour) for posterior sites.
The rates of fluid volume change during the initial part of the session before the rest period were very similar for some participants (e.g., in Figure 6 the arrows for participant 1 almost superimpose) and very different for other participants (e.g., in Figure 6 the arrows for participant 7 are dissimilar). When the rate of fluid volume change before the rest period was greater for Liquid-Out than Liquid-In, fluid volume retentions were comparable or greater for Liquid-Out at six anterior locations and at seven posterior locations, which represented six of seven cases for short-term and seven of seven cases for longterm (13 of 14 cases total) (Table 2). When the rate of fluid volume change before the rest period was greater for Liquid-In than Liquid-Out, fluid volume retentions were greater for Liquid-Out at four anterior locations and three posterior locations, which represented three of nine cases for short-term and four of nine cases for long-term (7 of 18 cases total) (Table 3). Fluid volume differences less than 0.10 percent were below the measurement sensitivity of the system and are represented in Table 2 with empty cells. 

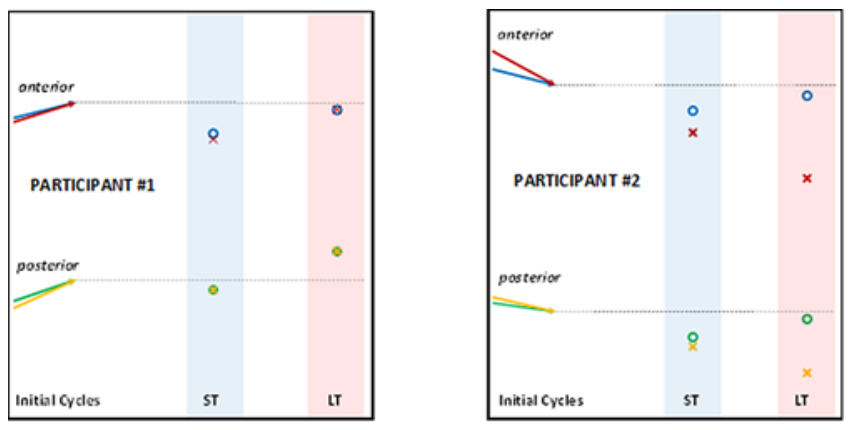

I Scale bar of $1.0 \%$ fluid volume change
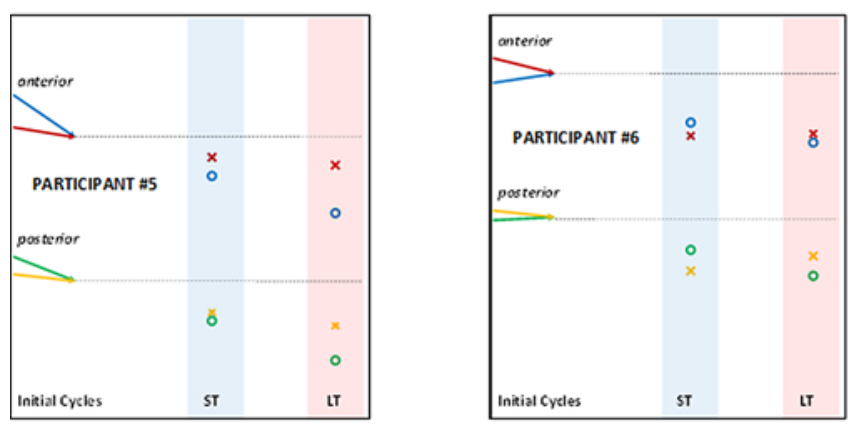
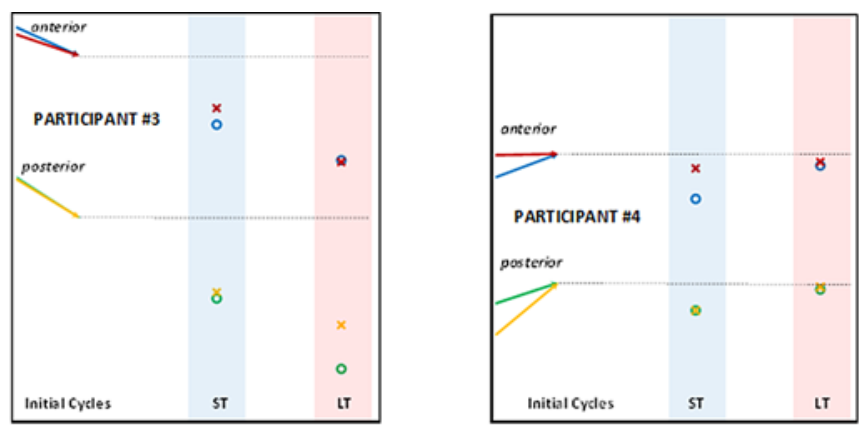

O Anterior Liquid-In $\times$ Anterior Liquid-Out O Posterior Liquid-In

$\times$ Posterior Liquid-Out

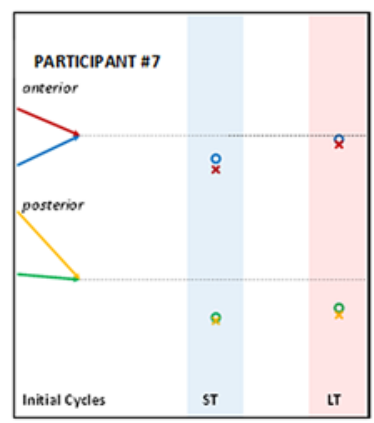

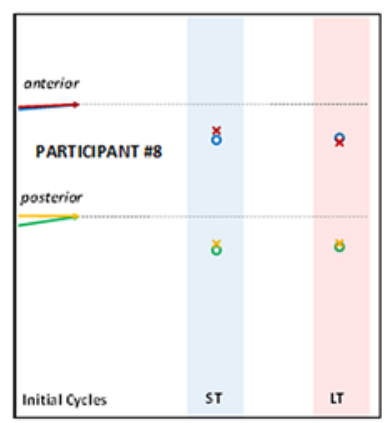

Figure 6.

Short-term and long-term fluid volume retentions and rates of fluid volume change before the rest period. Scales are the same for all figures. Horizontal axis is time. Vertical axis is percentage fluid volume change. Each black horizontal line is the $x$-axis (i.e., percentage fluid volume change of $0.0 \%$ ) for the corresponding data. Arrows show the rates of fluid volume change in the two cycles before the sitting period. Our sign convention is that a downward slope is more negative than a horizontal or upward slope. A steep negative slope is lower (more negative) than a shallow negative slope; a steep positive slope is higher (more positive) than a shallow positive slope. Short-term (ST) and long-term (LT) fluid volume retentions are shown in blue and red panels, respectively. See legend for location and condition.

\section{DISCUSSION}

A means to recover fluid into a prosthesis user's residual limb while sitting wearing the prosthesis may reduce diurnal fluid volume loss and enhance socket comfort. In this study, we temporarily released pressure of the prosthesis using a novel bladder-liner. We tested whether residual-limb fluid volume was recovered and then maintained during subsequent activity.

Results from this preliminary study demonstrate that removal of liquid from the bladder-liners during resting effectively enhanced fluid return to the posterior region of the residual limb. Differences in rest-period percentage posterior fluid volume change were statistically significant (Figure 5, Rest Period Recovery). This finding is consis- tent with our initial expectations. Vascular drive from the preceding walking activity, coupled with pressure relief on the residual limb during the rest period, likely reduced interstitial fluid pressures and enhanced arterialto-interstitial transport into the residual limb and/or reduced interstitial-to-venous transport out of the residual limb. The mean fluid volume gains here of $0.4 \pm 0.5$ percent anteriorly and $0.2 \pm 0.7$ percent posteriorly during the rest period are relatively low compared with results from a prior investigation, in which the socket was doffed during resting but the liner was left donned [15]. In that study, rest period fluid volume changes averaged $6.3 \pm 3.5$ percent for anterior and $6.1 \pm 4.7$ percent for posterior. There were several confounding variables that may have contributed to the lower magnitudes in the present study compared 
Table 2.

Differences between percentage fluid volume retentions (Liquid-Out Liquid In) when the rate of fluid volume change before the rest period was greater for Liquid-Out than Liquid-In. Fluid volume retentions were comparable or greater for Liquid-Out at six anterior locations and at seven posterior locations.

\begin{tabular}{lccc}
\hline Participant Location & $\begin{array}{c}\Delta \text { Short-Term } \\
\text { Fluid Volume } \\
\text { Retention (\%) }\end{array}$ & $\begin{array}{c}\Delta \text { Long-Term } \\
\text { Fluid Volume } \\
\text { Retention (\%) }\end{array}$ \\
\hline 1 & Ant & -0.17 & - \\
3 & Post & - & - \\
& Ant & 0.51 & - \\
4 & Post & 0.19 & 1.36 \\
5 & Post & - & - \\
& Ant & 0.58 & 1.50 \\
\hline${ }^{*}$ & Post & 0.27 & 1.09 \\
\hline
\end{tabular}

*Empty cells: difference $<0.1 \%$ resolution of system. Ant $=$ anterior, Post $=$ posterior.

Table 3.

Differences between percentage fluid volume retentions (Liquid-Out Liquid In) when the rate of fluid volume change before the rest period was greater for Liquid-In than Liquid-Out. Fluid volume retentions were greater for Liquid-Out at four anterior locations and three posterior locations.

\begin{tabular}{lccc}
\hline Participant Location & $\begin{array}{c}\Delta \text { Short-Term } \\
\text { Fluid Volume } \\
\text { Retention (\%) }\end{array}$ & $\begin{array}{c}\Delta \text { Long-Term } \\
\text { Fluid Volume } \\
\text { Retention (\%) }\end{array}$ \\
\hline 2 & Ant & -0.68 & -2.56 \\
4 & Post & -0.29 & -1.66 \\
6 & Ant & 0.95 & 0.11 \\
& Ant & -0.42 & 0.27 \\
7 & Post & -0.65 & 0.62 \\
& Ant & -0.34 & -0.16 \\
8 & Post & -0.10 & -0.22 \\
& Ant & 0.29 & -0.14 \\
& post & 0.20 & 0.11 \\
\hline
\end{tabular}

Ant $=$ anterior, Post $=$ posterior.

with the previous study. The approximately $25 \mathrm{~s}$ needed to remove liquid from the bladders at the outset of resting and associated delay in bioimpedance measurement in the present study may be partly responsible for the reduced fluid volume changes recorded. We noted previously that participants required less than $1 \mathrm{~min}$, on average, to reach approximately half the maximum restored fluid volume (when the socket was doffed but the liner maintained) [15]. The longer rest period in the prior study of $30 \mathrm{~min}$, instead of $10 \mathrm{~min}$ here, may also have contributed to different fluid volume changes over the rest period (the remainder of the activity protocol was identical between the studies). Finally, the custom liners used in the present study were stiffer than commercial products used in the previous investigation. Thus, the bladder-liners may have induced greater hoop stress and greater radial compression on the residual limb. Increased radial compression may have limited fluid volume recovery during resting in the present study. Though the posterior fluid volume gain differences between Liquid-In and Liquid-Out during rest were statistically significant, additional investigations to compare bladder-liner performance with other volume management strategies (e.g., temporary doffing) are needed.

Though removal of liquid from the bladder-liners during resting effectively enhanced fluid volume return to the posterior region of the residual limb, it did not do so for the anterior region of the limb. We suspect limb displacement within the socket during sitting partly explains why no differences were observed. When participants sat in the chair with their feet supported by the floor, their residual limb tended to pull away from the anterior surfaces of their socket, whether there was liquid in the bladders or not. Thus, we suspect there was not a meaningful difference in mechanical pressure applied anteriorly between the Liquid-In and Liquid-Out conditions. This interpretation is conjecture and would need to be evaluated through scientific investigation.

Though significantly greater posterior fluid volume gains were demonstrated during the rest period for Liquid-Out compared with Liquid-In, the gains were temporary. Differences in fluid volume retentions, both short term and long term, between Liquid-Out and Liquid-In were relatively low and not significantly different (Figure 5, Short-Term and Long-Term Retentions). Short-term fluid volume changes in a prior study, in which the socket was doffed and the liner left donned during resting, averaged $0.7 \pm 1.4$ percent for anterior and $0.1 \pm 1.5$ percent for posterior [15]. These values are greater than the present study's mean Liquid-Out results of $-1.1 \pm 0.5$ percent for anterior and $-1.2 \pm 0.6$ percent for posterior. Though the accommodation strategies used by participants (i.e., socket doffing versus bladder liquid removal) likely explain much of the difference, the duration of resting (i.e., $30 \mathrm{~min}$ in the prior study vs $10 \mathrm{~min}$ in the present study) and mechanical properties of liners used (i.e., custom here vs commercial liners used previously) may also have affected fluid retention. A further challenge is that, at present, it is unknown what fluid volume change is 
clinically relevant for most prosthesis users. In a related study, we demonstrated that a 1.0 percent change in socket volume was clinically detectable by an experienced practitioner evaluating socket fit [1]. A 1.0 percent socket volume change, roughly comparable to the fluid volume changes reported in the present investigation, corresponded to approximately a 1-ply sock change on an average-sized residual limb [22-23]. While these comparisons provide some insight into what affects short-term volume changes, it is clear that more extensive amputee participant studies need to be conducted over a day, a week, or longer to assess sensitivity to each variableaccommodation technique, duration of resting, and liner mechanical properties - and their relationship to participants' comfort. Such studies would facilitate understanding of the relative influence of each variable. Day-long studies would also likely result in greater percentage fluid volume changes than those measured in the approximately 30 min study reported here.

In the present study, day-to-day (i.e., intersession) differences in rates of fluid volume change before the start of the rest period were noted. Despite the consistent protocol, rates of fluid volume change before the rest period were not consistent over each participant's two test days. This finding is clinically relevant because it points to the need for emerging accommodation technologies to adjust to the user's individual day physiological status. Consistent rates of fluid volume change should not be assumed. The dayto-day variability may have affected rest and postrest limb fluid volume results in the present study.

If removal of liquid from the bladder-liner had a positive effect (i.e., limb fluid volume recovery and retention) or no effect (i.e., no limb fluid volume recovery and retention), we would expect greater short-term and long-term fluid volume retention for Liquid-Out when the rate of fluid volume change before the rest period was greater for Liquid-Out than Liquid-In. This result held for all but one case (Table 2). If maintaining liquid in the bladders had a positive effect or no effect, we would expect greater short-term and long-term fluid volume retention for Liquid-In when the rate of fluid volume change before the rest period was greater for Liquid-In than Liquid-Out. This was not the case, because in three of nine occurrences for short-term and four of nine occurrences for long-term ( 7 of 18 cases total), fluid volume retention was greater for Liquid-Out than Liquid-In (Table 3).

We conclude that the intervention tested in the present study, removing liquid from liquid-filled bladders within the liner during rest, may have benefitted fluid volume retention, but the effect was relatively low and not statistically significant. A bladder liner that could overcome the limitations identified in the present study may have a greater effect managing limb fluid volume. Because liner stiffness may contribute to the limited benefits demonstrated in this preliminary study, a next step should be to investigate bladder-liners that more easily stretch and allow for residual-limb fluid volume recovery during liquid release.

It is unclear at this point what percentage of limb fluid volume retention is clinically desirable to achieve optimal socket comfort and residual-limb health. To establish such metrics, studies relating fluid volume changes to socket comfort and satisfaction among a large number of prosthesis users are needed. Such information would be valuable to establishing guidelines for clinical care.

Another question worth pursuing is whether bladder liquid volumes can be periodically adjusted over a longer period (e.g., over the course of a day) to compensate for day-to-day changes in rates of limb fluid volume change. It is the authors' opinion that very small adjustments may be beneficial to limb health and maintenance of limb volume and may not be distracting to the prosthesis user. Incorporating small adjustments into an automated control system could relieve the need to manually adjust the prosthesis. However, if adjustments are large enough for the prosthesis user to be distracted and sense instability, then the benefits of automated socket adjustment would be lost. Characterization of day-to-day differences in rates of residual-limb fluid volume change on a large amputee participant population and its relationship to participant characteristics and activities would provide information helpful to developing an automated socket adjustment system.

The bladder-liners were well received by study participants and were durable for this relatively short study. The unsolicited positive comments from participants and the statistically significant benefits demonstrated posteriorly during rest with bladder liquid removed are encouraging in that the technology has the potential to improve comfort and satisfaction with the prosthesis.

\section{CONCLUSIONS}

A novel bladder-liner was tested on participants with transtibial limb loss to determine if releasing liquid from 
the bladders during a 10 min rest facilitated fluid volume recovery and retention, compared with not removing liquid. Results demonstrated that fluid volume recovery in the posterior region of the limb during rest was significantly improved, but retentions over the short term and long term were not significantly different. The high stiffness of the bladder-liner and differences in participants' physiological presentation between test sessions may have contributed to the lack of significant differences. The bladder-liners were well received by study participants. Further development of the bladder-liner technology to promote comfort and satisfaction with the prosthesis should be pursued.

\section{ACKNOWLEDGMENTS}

\section{Author Contributions:}

Study concept and design: J. E. Sanders, C. B. Redd, J. C. Cagle. Acquisition of data: C. B. Redd, D. Gardner, D. S. Harrison, K. J. Allyn, J. C. Cagle.

Analysis and interpretation of data: J. E. Sanders, B. J. Hafner, C. B. Redd.

Drafting of manuscript: J. E. Sanders.

Critical revision of manuscript for important intellectual content:

J. E. Sanders, B. J. Hafner, M. A. Ciol, K. J. Allyn, C. B. Redd,

J. C. Cagle, K. J. Allyn.

Statistical analysis: M. A. Ciol, B. J. Hafner.

Obtained funding: J. E. Sanders.

Financial Disclosures: The authors have declared that no competing interests exist.

Funding/Support: This material was based on work supported by the Department of Defense (grant W81XWH-10-1-1035) Congressionally-Directed Medical Research Program.

Additional Contributions: We appreciate assistance from Jason Wheeler, PhD, and Kevin Dullea at Sandia National Laboratories (Albuquerque, New Mexico) for creating the bladder-liners used in this research.

Institutional Review: All procedures were reviewed and approved by a University of Washington institutional review board. All participants signed informed consent forms.

Participant Follow-Up: The authors do not plan to inform participants of the publication of this study. However, participants have been encouraged to check the study Web site for updated publications.

\section{REFERENCES}

1. Sanders JE, Severance MR, Allyn KJ. Computer-socket manufacturing error: How much before it is clinically apparent? J Rehabil Res Dev. 2012;49(4):567-82.

[PMID:22773260]

http://dx.doi.org/10.1682/JRRD.2011.05.0097
2. Kosasih JB, Silver-Thorn MB. Sensory changes in adults with unilateral transtibial amputation. J Rehabil Res Dev. 1998;35(1):85-90. [PMID:9505256]

3. Sanders JE, Harrison DS, Allyn KJ, Myers TR, Ciol MA, Tsai EC. How do sock ply changes affect residual-limb fluid volume in people with transtibial amputation? J Rehabil Res Dev. 2012;49(2):241-56. [PMID:22773526] http://dx.doi.org/10.1682/JRRD.2011.02.0022

4. Street GM. Vacuum suspension and its effect on the limb. Orthop Tech. 2007;4:1-4.

5. Goswami J, Lynn R, Street G, Harlander M. Walking in a vacuum-assisted socket shifts the stump fluid balance. Prosthet Orthot Int. 2003;27(2):107-13. [PMID:14571940] http://dx.doi.org/10.1080/03093640308726666

6. Beil TL, Street GM. Comparison of interface pressures with pin and suction suspension systems. J Rehabil Res Dev. 2004;41(6A):821-8. [PMID:15685470] http://dx.doi.org/10.1682/JRRD.2003.09.0146

7. Board WJ, Street GM, Caspers C. A comparison of transtibial amputee suction and vacuum socket conditions. Prosthet Orthot Int. 2001;25(3):202-9. [PMID:11860094] http://dx.doi.org/10.1080/03093640108726603

8. Sanders JE, Harrison DS, Myers TR, Allyn KJ. Effects of elevated vacuum on in-socket residual limb fluid volume: Case study results using bioimpedance analysis. J Rehabil Res Dev. 2011;48(10):1231-48. [PMID:22234667] http://dx.doi.org/10.1682/JRRD.2010.11.0219

9. Gerschutz MJ, Denune JA, Colvin JM, Schober G. Elevated vacuum suspension influence on lower-limb amputee's residual limb volume at different vacuum pressure settings. J Prosthet Orthot. 2010;22(4):252-6. http://dx.doi.org/10.1097/JPO.0b013e3181f903df

10. Gerschutz MJ, Schober G, Denune JA, Colvin JM, Haynes ML. Elevated vacuum: residual limb skin's exposure to air pressure. In: Proceedings of the American Academy of Orthotists \& Prosthetists (AAOP) 39th Academy Annual Meeting and Scientific Symposium; 2013 Feb 20-23; Orlando, FL. Washington (DC): AAOP; 2013.

11. Traballesi M, Delussu AS, Fusco A, Iosa M, Averna T, Pellegrini R, Brunelli S. Residual limb wounds or ulcers heal in transtibial amputees using an active suction socket system. A randomized controlled study. Eur J Phys Rehabil Med. 2012;48(4):613-23. [PMID:22641248]

12. Ferraro C. Outcomes study of transtibial amputees using elevated vacuum suspension in comparison with pin suspension. J Prosthet Orthot. 2011;23(2):78-81. http://dx.doi.org/10.1097/JPO.0b013e3182173b83

13. Wernke M, Rink C, Powell HM, Gnyawali S, Kim J, Schroeder R. Quantification of residual limb skin health and circulation in response to elevated vacuum suspension. In: Proceedings of the American Academy of Orthotists \& Prosthetists (AAOP) 41st Academy Annual Meeting and 
Scientific Symposium; 2015 Feb 18-21; New Orleans, LA. Washington (DC): AAOP; 2015.

14. Klute GK, Berge JS, Biggs W, Pongnumkul S, Popovic Z, Curless B. Vacuum-assisted socket suspension compared with pin suspension for lower extremity amputees: Effect on fit, activity, and limb volume. Arch Phys Med Rehabil. 2011;92(10):1570-5. [PMID:21963124] http://dx.doi.org/10.1016/j.apmr.2011.05.019

15. Sanders JE, Hartley TL, Phillips RH, Ciol MA, Hafner BJ, Allyn KJ, Harrison DS. Does temporary socket removal affect residual limb fluid volume of trans-tibial amputees? Prosthet Orthot Int. 2016;40(3):320-8. [PMID:25710944] http://dx.doi.org/10.1177/0309364614568413

16. Sandia National Laboratories. Dynamic Socket [Internet]. Albuquerque (NM): Sandia National Laboratories; 2016 [cited 2014 Jun 4]. Available from: http://www.sandia.gov/ research/robotics/cybernetics/dynamic socket.html

17. Sanders JE, Lam D, Dralle AJ, Okumura R. Interface pressures and shear stresses at thirteen socket sites on two persons with transtibial amputation. J Rehabil Res Dev. 1997; 34(1):19-43. [PMID:9021623]

18. Sanders JE, Moehring MA, Rothlisberger TM, Phillips RH, Hartley T, Dietrich CR, Redd CB, Gardner DW, Cagle JC. A bioimpedance analysis platform for amputee residual limb assessment. IEEE Trans Biomed Eng. 2016;63(8): 1760-70. [PMID:26595906] http://dx.doi.org/10.1109/TBME.2015.2502060

19. De Lorenzo A, Andreoli A, Matthie J, Withers P. Predicting body cell mass with bioimpedance by using theoretical methods: A technological review. J Appl Physiol. 1997; 82(5):1542-58. [PMID:9134904]

20. Fenech M, Jaffrin MY. Extracellular and intracellular volume variations during postural change measured by segmental and wrist-ankle bioimpedance spectroscopy. IEEE
Trans Biomed Eng. 2004;51(1):166-75. [PMID:14723506] http://dx.doi.org/10.1109/TBME.2003.820338

21. Rothman KJ. No adjustments are needed for multiple comparisons. Epidemiology. 1990;1(1):43-6. [PMID:2081237] http://dx.doi.org/10.1097/00001648-199001000-00010

22. Cagle JC, D’Silva KJ, Hafner BJ, Harrison DS, Sanders JE. Amputee socks: Sock thickness changes with normal use. Prosthet Orthot Int. 2016;40(3):329-35. [PMID:25733408] http://dx.doi.org/10.1177/0309364614568412

23. Fernie GR, Holliday PJ. Volume fluctuations in the residual limbs of lower limb amputees. Arch Phys Med Rehabil. 1982;63(4):162-5. [PMID:7082139]

Submitted for publication December 25, 2014. Accepted in revised form January 25, 2016.

This article and any supplementary material should be cited as follows:

Sanders JE, Redd CB, Cagle JC, Hafner BJ, Gardner D, Allyn KJ, Harrison DS, Ciol MA. Preliminary evaluation of a novel bladder-liner for facilitating residual-limb fluid volume recovery without doffing. J Rehabil Res Dev. 2016;53(6):1107-20.

http://dx.doi.org/10.1682/JRRD.2014.12.0316

ORCID: Joan E. Sanders, PhD: E-8204- 2011; Brian J. Hafner, PhD: M-6322-2013; Marcia A. Ciol: 0000-00016406-4706

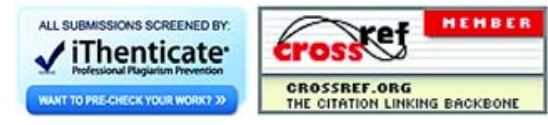

